\title{
Hábitos alimentarios del pez triglido Prionotus ruscarius (Gilbert \& Starks, 1904) durante 1996, en las costas de Jalisco y Colima, México
}

\author{
Feeding habits of the triglid fish Prionotus ruscarius (Gilbert \& Starks, 1904) \\ during 1996, on the Jalisco and Colima coast, México \\ Alma Rosa Raymundo-Huizar' ${ }^{1}$ y Mirella Saucedo Lozano² \\ ${ }^{1}$ Universidad de Guadalajara. Centro Universitario de la Costa. Departamento de Ciencias Médicas y Biológicas. \\ Av. Universidad \#203. Delegación Ixtapa. Puerto Vallarta 48280. Jalisco, México \\ ${ }^{2}$ Universidad de Guadalajara. Centro Universitario de la Costa Sur. Departamento de Estudios para el Desarrollo \\ Sustentable de Zonas Costeras. Gómez Farias \#82, San Patricio-Melaque 48980. Jalisco, México \\ arhuizar@pv.udg.mx
}

\begin{abstract}
The stomach content of the common searobin Prionotus ruscarius is described and analysed in this study. A total of 128 specimens, were captured from the continental shelf of Jalisco and Colima, Mexico, between January and December 1996. Percentages of numeric, gravimetric, frequency of occurrence and index of relative importance (IIR) were calculated for each prey item. Thirty food items were observed in the diet. The index of relative importance (IIR) showed that $P$. ruscarius mainly prey on penaeids shrimp (45.5\%), brachyuran (37.8\%) and stomatopods (9.7\%). Significant feeding variations were found in different fish body sizes. According to Levins Index, used to determine the trophic spectrum, $P$. ruscarius had a medium grade of specialization. Penaeid shrimps and brachyurans were observed as secondary food items, based on the feeding index.
\end{abstract}

Key words: Triglidae, continental shelf, stomach content
Resumen.- Este estudio describe y analiza el contenido estomacal del pez triglido Prionotus ruscarius. Un total de 128 ejemplares fueron capturados en la plataforma continental de Jalisco y Colima, México, de enero a diciembre de 1996. Se calculó los porcentajes numérico, gravimétrico, de frecuencia de ocurrencia y del índice de importancia relativa (IIR) para cada uno de los ítems presa. Se determinó que la dieta de $P$. ruscarius estuvo constituida por 30 ítems alimentarios. De acuerdo con el IIR, esta especie se alimenta preferentemente de camarones peneidos (45,5\%), braquiuros (37,8\%) y estomatópodos (9,7\%). Se encontró una variación en la alimentación a diferentes longitudes corporales de los ejemplares analizados y en distintos meses de muestreo. Mediante el índice de Levins se determinó el espectro trófico de $P$. ruscarius el que indicó que este pez presentaba un grado medio de especialización. Los camarones peneidos y los braquiuros se observaron como alimento secundario, según el índice alimentario.

Palabras clave: Triglidae, plataforma continental, contenido estomacal

contribuyan a una correcta administración de los recursos pesqueros. No solo es importante generar este tipo de conocimiento para especies de valor económico, sino también para todas aquellas con las que se relaciona ecológicamente, ya que una alteración en su dinámica podría afectar directa o indirectamente la supervivencia de cualquier especie.

Los peces de la familia Triglidae son comunes en zonas templadas y tropicales, existiendo aproximadamente 100 especies. Estos peces habitan en las plataformas y taludes continentales e insulares, encontrándose hasta los $180 \mathrm{~m}$ de profundidad (Bussing 1995). Existen pocos estudios sobre la alimentación de los peces de esta familia, y en particular para Prionotus ruscarius (Gilbert \& Starks, 1904). Si bien $P$. ruscarius, no es una especie objeto de 
las pesquerías comerciales, aparece con frecuencia en las capturas con redes de arrastre, a veces, en grandes cantidades, por lo que constituye un elemento importante en el ecosistema que habita. Es así que el objetivo del presente estudio es realizar un análisis cualitativo y cuantitativo de la alimentación de $P$. ruscarius, obtenidos en la costa de Jalisco y Colima, México. Además, se determina si existen variaciones ontogenéticas y anuales en la alimentación de este pez.

De los antecedentes alimentarios para peces triglidos se encuentran los estudios de Ross $(1977,1978)$ quien analizó la repartición de recursos alimenticios de estos peces en el Golfo de México e investigó las variaciones ontogenéticas de P. scitulus. Braga \& Braga (1987), por su parte, estudiaron los hábitos alimentarios de $P$. punctatus, Schmitter-Soto \& Castro-Aguirre (1996) analizaron los hábitos alimenticios de P. stephanophrys, Bellator gymnostethus y P. albirostris en la costa del Pacífico de Baja California Sur. No existen estudios de la dieta de $P$. ruscarius en México

\section{Material y métodos}

Los ejemplares de $P$. ruscarius fueron capturados sobre la plataforma continental de Jalisco y Colima, en un área comprendida desde la desembocadura del río Cuitzmala, en Punta Farallón estado de Jalisco ( $\left.19^{\circ} 22^{\prime} \mathrm{N}, 105^{\circ} 01^{\prime} \mathrm{O}\right)$ como límite norte, hasta Cuyutlán, en el estado de Colima $\left(18^{\circ} 55^{\prime} \mathrm{N}, 104^{\circ} 08^{\prime} \mathrm{W}\right)$ al sur (Fig. 1).

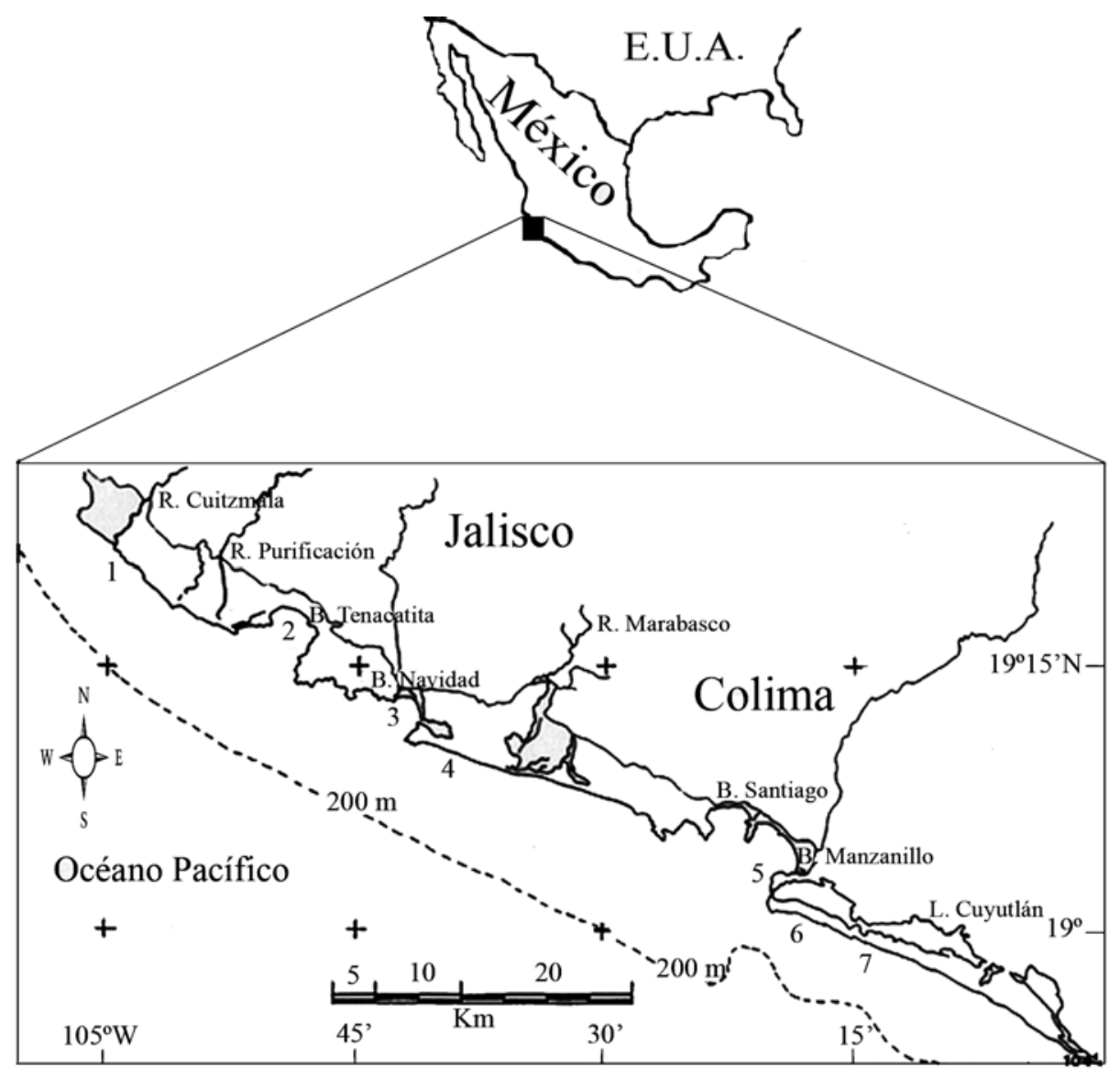

Figura 1

Área de estudio y localización de los sitios de muestreo. (1) Cuitzmala, (2) Tenacatita, (3) Bahía de Navidad, (4) El Coco, (5) Manzanillo-Santiago, (6) Tepalcates, (7) Cuyutlán

Study area and sampling location. (1) Cuitzmala, (2) Tenacatita, (3) Bahia de Navidad, (4) El Coco,

(5) Manzanillo-Santiago, (6) Tepalcates, (7) Cuyutlan 
Las muestras de peces y su contenido estomacal de $P$. ruscarius fueron recolectados a bordo del barco BIP-V del Departamento de Estudios para el Desarrollo Sustentable de Zonas Costeras de la Universidad de Guadalajara, desde enero a diciembre de 1996. Las muestras fueron obtenidas en arrastres nocturnos correspondientes a tres campañas de muestreo denominadas 'demersales' (marzo, junio y noviembre de 1996). Se efectuaron arrastres en siete sitios de muestreo, definiendo cuatro estratos batimétricos (I: 20 m; II: 40 m; III: 60 m y IV: 80 m de profundidad media); además se llevaron a cabo muestreos mensuales (enero, abril, mayo, julio, agosto, octubre y diciembre de 1996) realizados en Bahía Navidad, Jalisco y Playa el Coco, Colima, a profundidades de 20 y $60 \mathrm{~m}$, siguiendo la misma metodología de las campañas. En cada uno de los estratos se realizaron lances de pesca de 30 minutos, utilizando redes de babor y estribor simultáneamente, a una velocidad constante de dos nudos. Durante febrero y septiembre no fue posible la obtención de muestras debido a condiciones climáticas adversas y problemas mecánicos del barco.

Para cada organismo se registró la longitud corporal total (LT) (desde la parte anterior del rostro hasta el punto posterior de la aleta caudal), utilizando un ictiómetro convencional de $\pm 1 \mathrm{~mm}$ de precisión, mientras que el peso corporal individual se determinó mediante una balanza semianalítica de $\pm 0,1 \mathrm{~g}$. Posteriormente, se extrajeron y recolectaron los estómagos de cada ejemplar en bolsas de plástico, debidamente etiquetadas, conteniendo una solución de formaldehído al 10\%. El contenido de cada estómago fue analizado con la ayuda de un microscopio estereoscópico. Los diversos componentes de la dieta fueron identificados mediante claves especializadas, hasta el nivel taxonómico más bajo posible, en función del grado de digestión en que se encontraban los organismos. Para la identificación de los crustáceos se consultaron Rathbun (1930), Garth (1958), Rodríguez De la Cruz (1987), Hendrickx \& Salgado-Barragán (1991), Hendrickx (1996) y Landa-Jaime et al. (1997) y para los peces, Jordan \& Evermann (1896-1900), Castro-Aguirre (1978), Thomson et al. (1979), Allen \& Robertson (1994) y las guías de la FAO (Fischer et al. 1995).

Se calcularon los índices: numérico $(\mathrm{N})$, gravimétrico (P), porcentaje gravimétrico según Rosecchi \& Nouaze (1987) y de frecuencia de ocurrencia (F). Posteriormente se calculó el índice de importancia relativa (IIR) de Pinkas et al. (1971), en su versión modificada por Hacunda (1981), quien reemplazó el volumen por el peso.

El análisis del espectro trófico respecto a las variaciones mensuales y diferentes clases de talla, se realizó utilizando el porcentaje del peso de cada presa.
Con los valores de la longitud total de $P$. ruscarius se determinó el número de intervalos de talla; para los intervalos de clase se utilizó la fórmula de Sturges (1926): $\mathrm{k}=1+3,322\left(\log _{10} \mathrm{n}\right)$, donde, $\mathrm{k}=$ número de intervalos de clase, $\mathrm{n}=$ número total de valores.

Una vez obtenido el número de intervalos de clase para cada especie, se procedió a determinar la amplitud de este intervalo, el cual se calculó con la siguiente fórmula (Daniel 1997): $\mathrm{W}=\mathrm{R} / \mathrm{k}$, donde, $\mathrm{W}=$ amplitud del intervalo, $\mathrm{R}=$ valor máximo menos el valor mínimo.

Esta aproximación al número y amplitud de intervalos de talla ha sido aplicado anteriormente en otras especies (Bocanegra-Castillo et al. 2000, Kouamélan et al. 2000), considerándose apropiado para explicar variaciones en la dieta respecto de la talla.

Se realizó una evaluación de la amplitud del espectro trófico (amplitud de nicho) con el fin de determinar el grado de especialización en la dieta de $P$. ruscarius. Para tal efecto se utilizó la medida de Levins (1968), estandarizada por el método de Hespenheiden (1975), utilizando una escala de 0 a 1 , considerándose como especialista cuando el valor es cercano a cero y generalista cuando el valor es cercano a 1 (Krebs 1989).

$$
B=\frac{1}{\mathrm{n}} B_{\substack{P j^{2} \\ \mathrm{i}=1}} B_{a} \frac{B-1}{n-1}
$$

donde, B es la medida de Levins sobre la amplitud de la dieta; $\mathrm{B}_{\mathrm{a}}$ la medida de Levins estandarizada; $P j$ equivale a la proporción con la cual cada categoría de presa contribuye a la dieta y $n$ es el número total de recursos alimentarios.

Se estableció el grupo de presas de mayor importancia en la dieta, así como aquellos que son consumidos de manera accidental, con el índice alimentario (IA) (Lauzanne 1975), modificado: IA= \%F ×\%P (\%A)/100.

$\mathrm{Al}$ relacionar las preferencias alimentarias (\%F) y la importancia relativa de las presas en peso (\%P) o área (\%A), este índice reúne los ítems alimentarios en una función que permite distinguir al alimento preferencial de aquel cuyo consumo es de baja frecuencia (Rosecchi \& Nouaze 1987 modificado). Los valores de IA varían de 0 a 100\% y se categorizan en función de su importancia relativa como: 1) alimento preferencial (AI > 50), 2) secundario $(25<$ IA $>50)$, frecuente $(10<$ IA $>25)$ y 4$)$ accidental (IA < 10) (Lauzanne 1975). 
Tabla 1

Composición y descripción numérica del espectro alimentario de Prionotus ruscarius de la costa de Jalisco y Colima, México. Peso (P), frecuencia de ocurrencia (F), número (N), índice de importancia relativa (IIR) de las presas e índice alimentario (IA)

Composition and numerical description of the dietary spectrum of Prionotus ruscarius from Jalisco and Colima coasts, Mexico. Weight (P), frequency of occurrence $(\mathrm{F})$, number $(\mathrm{N})$, index of relative importance (IIR) for each prey item and feeding index (IA)

\begin{tabular}{|c|c|c|c|c|c|c|}
\hline \multicolumn{2}{|c|}{ Componentes alimentarios } & $\% P$ & $\% F$ & $\% N$ & $\% I I R$ & $I A$ \\
\hline \multirow[t]{11}{*}{ BRAQUIUROS } & Braquiuros & 1,04 & 6,31 & 33,33 & 10,65 & \\
\hline & Portúnido & 10,40 & 14,41 & 9,56 & 16,63 & \\
\hline & Portunus sp. & 4,80 & 4,50 & 1,91 & 1,75 & \\
\hline & P. xantusi & 12,22 & 5,41 & 2,19 & 4,50 & \\
\hline & P. afinis & 5,80 & 5,41 & 1,64 & 2,32 & \\
\hline & Euphylax dobii & 0,54 & 0,90 & 0,27 & 0,04 & \\
\hline & Euphylax sp. & 0,77 & 1,80 & 0,55 & 0,14 & \\
\hline & Arenaeus mexicanus & 6,26 & 3,60 & 1,64 & 1,65 & \\
\hline & Raninoides benedecti & 0,18 & 0,90 & 0,27 & 0,02 & \\
\hline & Persephonasp. & 0,01 & 0,90 & 0,27 & 0,02 & \\
\hline & Total & 42,03 & 44,14 & 51,64 & 37,72 & 18,85 \\
\hline \multirow{8}{*}{$\begin{array}{l}\text { CAMARONES } \\
\text { PENEIDOS }\end{array}$} & Trachypenaeus sp. & 0,92 & 1,80 & 0,55 & 0,15 & \\
\hline & T. pacificus & 4,20 & 6,31 & 10,38 & 5,32 & \\
\hline & T. brevisuturae & 8,11 & 8,11 & 5,19 & 6,23 & \\
\hline & Sycionia sp. & 3,79 & 9,91 & 3,01 & 3,89 & \\
\hline & S. disdorsalis & 15,74 & 16,22 & 7,10 & 21,41 & \\
\hline & Metapenaopsis beebei & 0,24 & 0,90 & 0,27 & 0,03 & \\
\hline & Restos de camarón & 7,14 & 20,72 & 0,00 & 8,55 & \\
\hline & Total & 40,13 & 63,96 & 26,50 & 45,58 & 25,67 \\
\hline \multirow[t]{5}{*}{ ESTOMATÓPODOS } & Estomatópodos & 2,27 & 9,01 & 3,01 & 2,74 & \\
\hline & Eurysquilla veleronis & 0,03 & 0,90 & 0,27 & 0,02 & \\
\hline & Squilla hancocki & 10,31 & 8,11 & 3,83 & 6,63 & \\
\hline & Meiosquilla swetii & 0,21 & 1,80 & 0,55 & 0,08 & \\
\hline & Total & 12,82 & 19,82 & 7,65 & $\mathbf{9 , 4 7}$ & 2,54 \\
\hline \multirow{5}{*}{$\begin{array}{l}\text { OTROS } \\
\text { CRUSTÁCEOS }\end{array}$} & Microcrustáceos & 1,04 & 7,21 & 9,29 & 4,48 & \\
\hline & Larvas de crustáceos & 0,28 & 1,80 & 1,09 & 0,15 & \\
\hline & Carideo & 0,06 & 1,80 & 0,82 & 0,09 & \\
\hline & Cumáceos & 0,06 & 0,90 & 1,91 & 0,11 & \\
\hline & Total & 1,45 & 11,71 & 13,11 & 4,83 & 0,17 \\
\hline \multicolumn{2}{|c|}{ RESTOS DE CRUSTÁCEOS } & 3,13 & 13,51 & 0,00 & 2,35 & 0,42 \\
\hline \multirow[t]{5}{*}{ PECES } & Pleuronectiformes & 0,03 & 0,90 & 0,27 & 0,02 & \\
\hline & Sinphunus sp. & 0,24 & 1,80 & 0,55 & 0,08 & \\
\hline & Syaciun latifrons & 0,06 & 1,80 & 0,27 & 0,04 & \\
\hline & Restos de peces & 0,12 & 1,80 & 0,00 & 0,01 & \\
\hline & Total & 0,45 & 6,31 & 1,09 & 0,15 & 0,03 \\
\hline
\end{tabular}




\section{Resultados}

Un total de 128 ejemplares de $P$. ruscarius fueron capturados a profundidades de 20, 40 y 60 m, desde enero a diciembre de 1996. El rango de longitudes corporales varió entre 7,2 y $38 \mathrm{~cm}$ mientras quel peso corporal fluctuó entre 5 y 770 g.

El 92\% de la muestra total tenía contenido estomacal. De ellos, se encontró un espectro trófico constituido por 30 componentes alimentarios (Tabla 1), que para fines de la descripción general de la dieta, se agruparon en seis categorías principales: braquiuros, camarones peneidos, estomatópodos, otros crustáceos, restos de crustáceos y peces. A partir de estas categorías se identificó un total de 13 especies, seis géneros y algunos quedaron solo como restos de peces, restos de camarón y larvas de crustáceos.

El principal componente alimentario de $P$. ruscarius fue el grupo de los camarones peneidos ( $\mathrm{N}=26,50 \%, \mathrm{P}$ $=40,13 \%, \mathrm{~F}=63,96 \%$, IIR $=45,58 \%$ ), de los cuales la presa más importante fue Sycionia disdorsalis. El siguiente grupo en orden de importancia lo constituyeron los braquiuros, donde los portunidos fueron las presas más frecuentes. En el grupo de los estomatópodos, la presa más importante fue Squilla hancocki. El grupo de los otros crustáceos presentó porcentajes bajos, al igual que el de restos de crustáceos y peces (Fig. 2).

Se observaron algunas variaciones en el análisis mensual del espectro alimentario. En cuanto al porcentaje en peso, los camarones peneidos fueron los más importantes en enero, marzo y noviembre (73,5\%, 48\% y 71,5\%, respectivamente); los braquiuros fueron un grupo importante en abril, mayo, junio y diciembre (52\%, 47,2\%, 85\% y 61,2\%, respectivamente). Cabe destacar la presencia de porcentajes importantes de otros grupos, como los estomatópodos en abril (26\%) y junio (53,7\%), así como los restos de crustáceos en agosto (34,2\%) y noviembre (22,6\%); sin embargo, el grupo de 'otros crustáceos' solo tuvo un porcentaje importante en agosto (20\%) (Fig. 3).

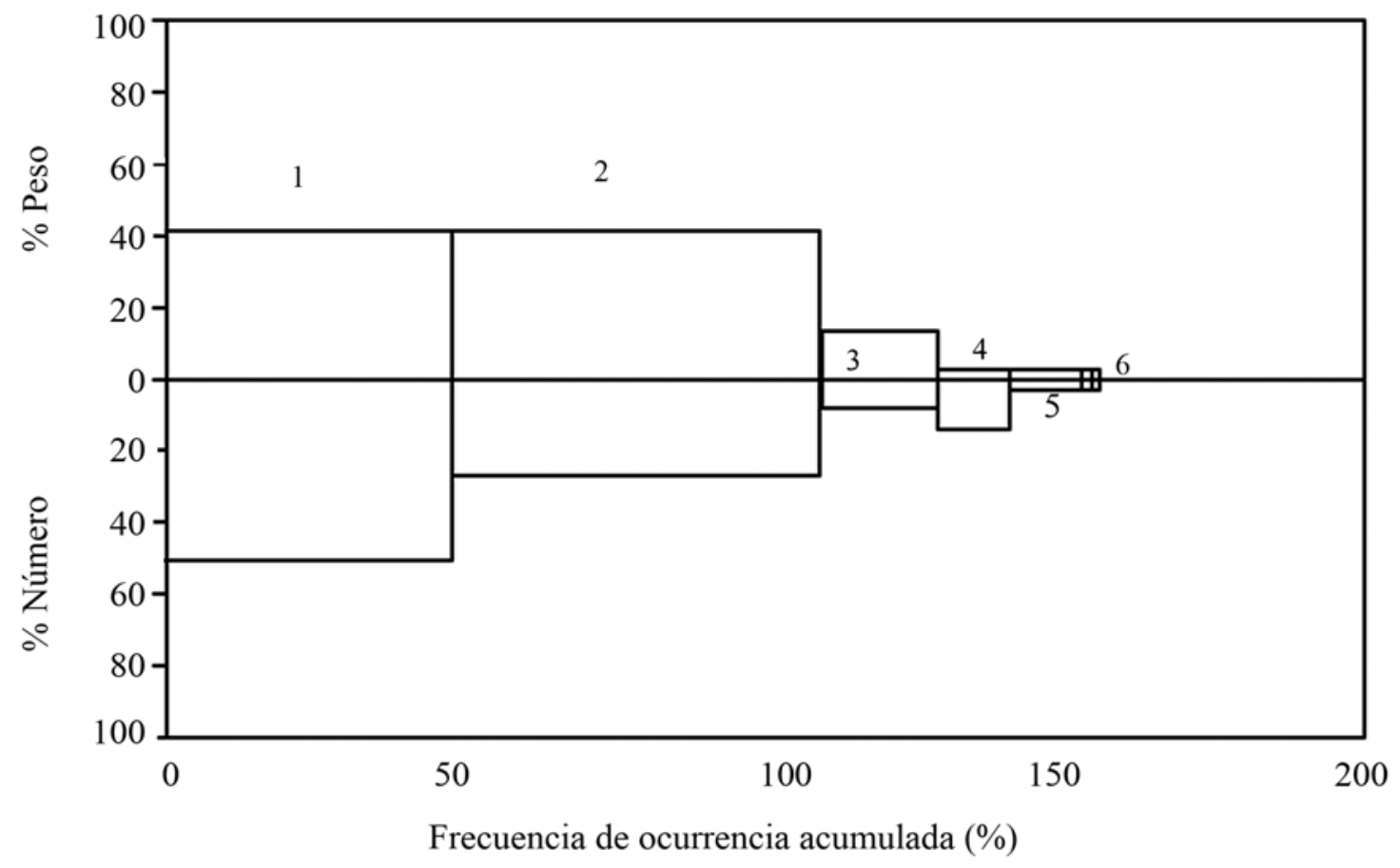

Figura 2

Índice de importancia relativa de los principales grupos de presas de Prionotus ruscarius, durante el periodo de estudio. 1) Braquiuros, 2) camarones peneidos, 3) estomatópodos, 4) otros crustáceos, 5) restos de crustáceos, 6) peces

Index of relative importance of the main prey groups of Prionotus ruscarius during the study period. 1) Brachyurans, 2) peneid shrimps, 3) stomatopods, 4) other crustaceans 5) crustacean remains, 6) fish 


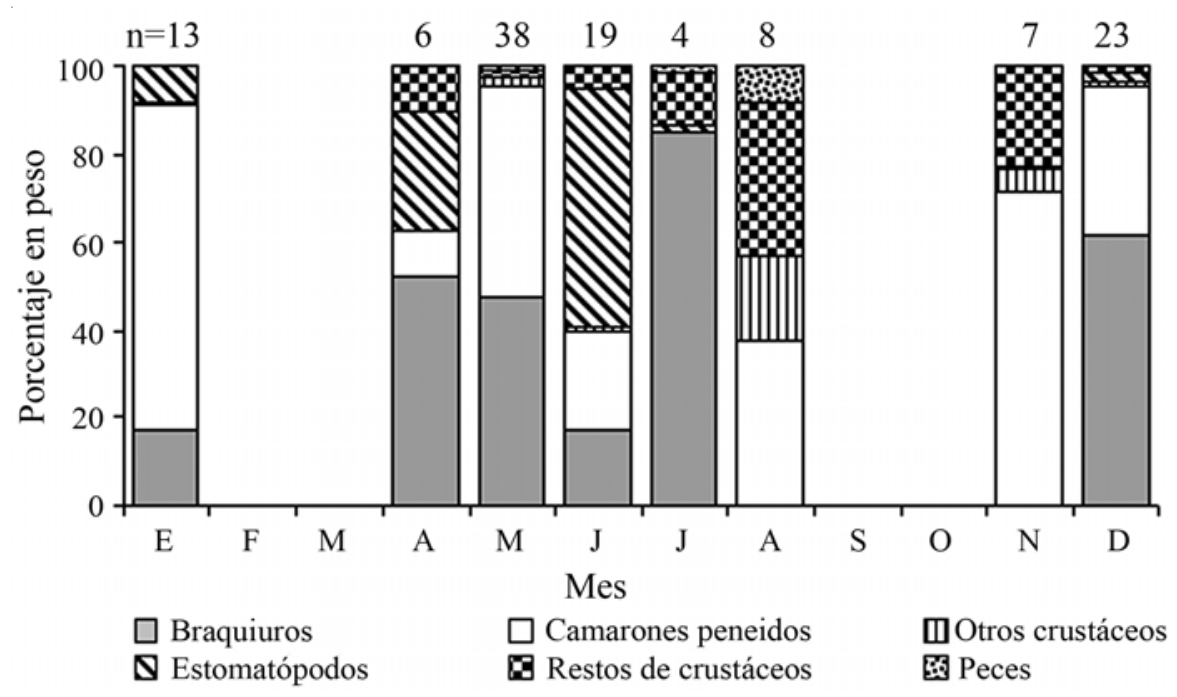

Figura 3

Porcentaje en peso (g) de los grupos de presa de Prionotus ruscarius en 8 meses de muestreo. Sobre las barras se indica el número de estómagos analizados

Weight (g) percentage of prey groups of Prionotus ruscarius in 8 sampling months. Above the bars are indicated the number of stomachs analyzed

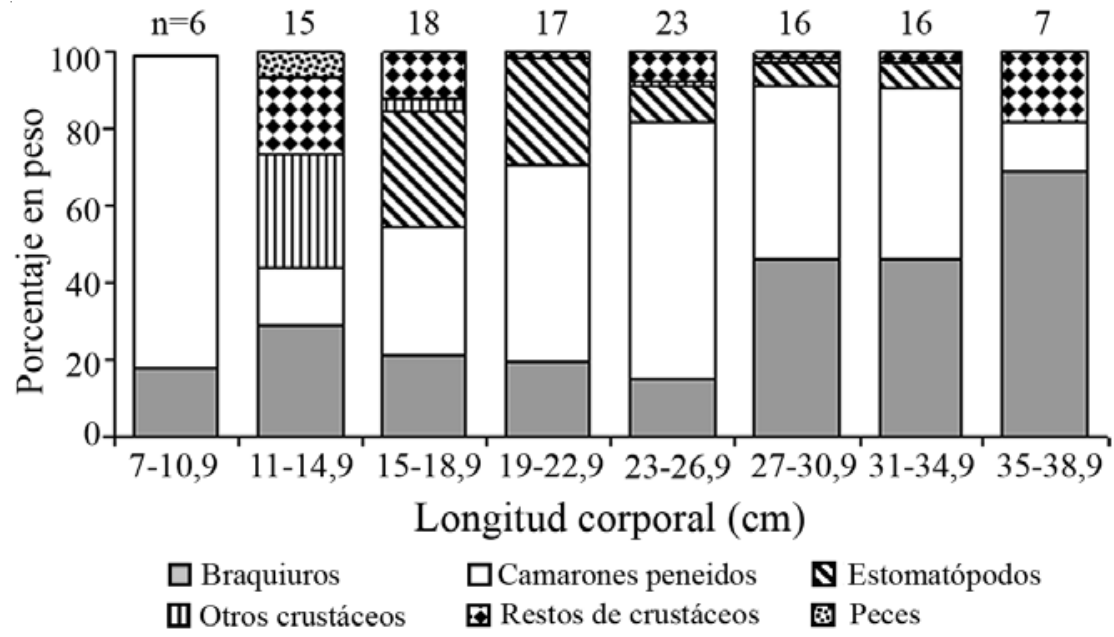

Figura 4

Porcentaje en peso (g) de los grupos de presas en 8 categorías de longitud corporal de Prionotus ruscarius. Sobre las barras se indican número de estómagos analizados

Weight (g) percentage of prey groups in 8 catogories of fish body length of Prionotus ruscarius. Above the bars are indicated the number of stomachs analyzed 
Los resultado del análisis ontogénico de la dieta, basado en ocho clases de talla (Fig. 4), muestran que los organismos pequeños $(7-10,9 \mathrm{~cm} \mathrm{LT})$ tiene preferencia alimentaria sobre los camarones peneidos $(\mathrm{P}=82 \%)$ y braquiuros ( $\mathrm{P}=18 \%)$. Para la clase de 11 a $14,9 \mathrm{~cm} \mathrm{LT}$, la dieta fue variada, incluyendo a los braquiuros (29\%), otros crustáceos (30\%), estomatópodos (16\%) y restos de crustáceos (18\%). La clase de 15-18,9 cm y 19-22,9 cm LT, consumen principalmente estomatópodos (33 y $51 \%$, respectivamente) y camarones peneidos (30 y 28\%, respectivamente) y la clase de talla de 23 a $26,9 \mathrm{~cm}$ LT, consume, en su mayoría, estomatópodos (67\%); sin embargo, se observó una disminución en el consumo de éstos conforme aumentó la talla de los ejemplares y, contrariamente, se incrementó la presencia de braquiuros (clases de talla 27-30,9 cm, 31-34,9 cm y 35-38,9 cm LT).

Esta especie mostró una estrecha amplitud de nicho para los meses analizados $(<0,5)$, con un valor del índice de Levins de 0,15 (Fig. 5) como máximo para el mes de noviembre.

De acuerdo al índice alimentario (IA), los grupos que constituyen el espectro trófico de $P$. ruscarius, como los braquiuros $(18,5 \%)$ y los camarones peneidos $(25,7 \%)$, fueron considerados dentro de la categoría de alimento secundario; por su parte el grupo de estomatópodos (2,5\%), otros crustáceos $(0,2 \%)$, restos de crustáceos $(0,4 \%)$ y peces $(0,02 \%)$, fueron considerados como alimento accidental.

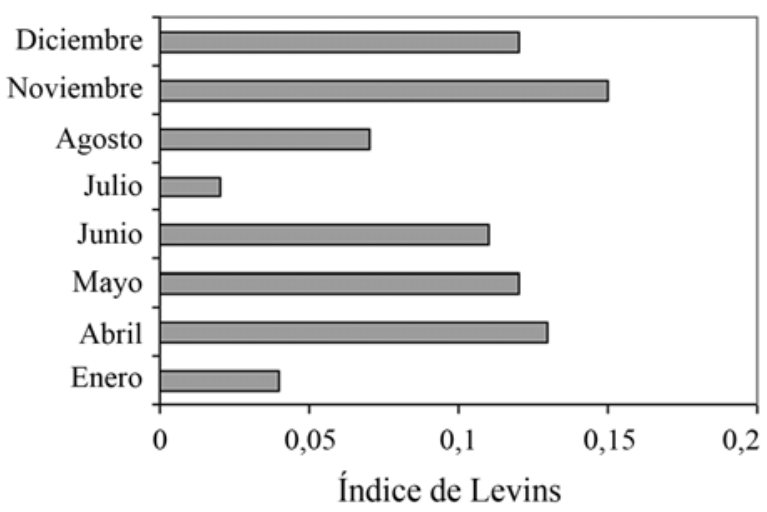

Figura 5

\section{Diversidad trófica mensual de Prionutus ruscarius}

Monthly trophic diversity of Prionutus ruscarius

\section{Discusión}

A pesar de realizar un esfuerzo de muestreo similar en todos los meses y en todos los estratos de profundidad, en este estudio, los ejemplares de $P$. ruscarius aparecieron únicamente en ocho meses (enero, abril, mayo, junio, julio, agosto, noviembre y diciembre) y fueron capturados en todos los casos en profundidades de 20, 40 y $60 \mathrm{~m}$. Esto permite suponer que $P$. ruscarius se encuentra en zonas someras de la plataforma o cercanos a la costa, de acuerdo a los siguientes antecedentes: Yáñez-Arancibia (1978) indica que esta especie es un visitante ocasional de lagunas costeras o estuarios; Schmitter-Soto (1992) encontró a esta especie hasta $70 \mathrm{~m}$ de profundidad pero en cantidades muy bajas y menciona además que prefieren las aguas protegidas de bahía Magdalena que la plataforma abierta. Por su parte, Bussing (1995) encuentra que los triglidos habitan en las plataformas y taludes continentales e insulares; de igual forma, Schmitter-Soto \& Castro-Aguirre (1996) mencionan que P. ruscarius fue encontrada en el complejo lagunar Magdalena-Almejas. Así mismo, Mariscal-Romero et al. (1998) encuentran a $P$. ruscarius entre las 32 especies más abundantes de peces demersales en la plataforma continental de Jalisco y Colima, México y a profundidades no mayores de 60 metros.

Aunque este estudio constituye uno de los primeros aportes a la biología trófica de $P$. ruscarius, se puede apreciar que los estudios realizados por otros autores para especies de la misma familia, como el de Reid (1954) y el de Springer \& Woodburn (1960) para P. scitulus, encuentran pequeños crustáceos y poliquetos como presas más importantes; Ross (1978) menciona para Branchiostoma floridae que el $61 \%$ del peso está representado por céfalocordados y que, numéricamente, los cumáceos fueron los dominantes con un $40 \%$ del total de las presas. Por su parte, Yañez-Arancibia (1978) encuentra que $P$. ruscarius es un consumidor de tercer orden (carnívoro) y que la presencia de vegetales y detritus es accidental; Braga \& Braga (1987) mencionan que la alimentación de $P$. punctatus está constituida básicamente de crustáceos destacándose los decápodos natantia y reptantia, isópodos, cumáceos, anfípodos y ocasionalmente, algunos peces. A pesar que SchmitterSoto (1992) sólo analizó un estómago, encontró que $P$. ruscarius se alimenta de miscidáceos, estomatópodos (Schmittius polita), peneidos (Sicyonia ingentis) y braquiuros (Panopeus purpureus), dominando este último por volumen y abundancia. Todo lo anterior permite mencionar que existen similitudes con los resultados obtenidos en el presente trabajo ya que si bien no se encontraron las mismas especies, sí fueron ejemplares 
Vol. 43, Nº 1,2008

pertenecientes a las mismas familias y en porcentajes similares, de igual forma tanto en los trabajos anteriores como en el presente, las presas principales fueron los crustáceos.

Se han reportado cambios ontogénicos en la dieta de otras especies de la familia Triglidae, como lo menciona el trabajo de Ross (1977) para P. scitulus, donde observó que los juveniles ingieren principalmente epifauna y los adultos, infauna; de igual forma para la familia Triglidae, Moreno \& Amich (1988) atribuyen los cambios de dieta a la talla de reclutamiento y maduración. De tal manera que con esto se puede observar que las diferencias tróficas corresponden a la transición de tamaño entre peces inmaduros y maduros. Estos cambios se pueden considerar como una característica general en la alimentación de los peces, pero algunas veces resultan ser poco claras.

Por lo indicado, se sugiere que la especie está adaptada para soportar exitosamente las variaciones estacionales que presentan las poblaciones de las presas disponibles en el ambiente. Así, existe un cambio en las preferencias alimentarias, relacionado con la abundancia relativa de las especies consumidas. Al parecer, la dieta está constituida más por la disponibilidad de presas que por alguna preferencia en particular.

A pesar que $P$. ruscarius presenta un número considerable de componentes alimentarios (30), se observó una amplitud baja (menor de 0,5). Esto posiblemente es debido a las diferencias en la abundancia y disponibilidad de los ítems que constituyen la base alimentaría de los depredadores, aun cuando se puede observar en la dieta de esta especie, una preferencia por braquiuros y camarones peneidos, que fueron considerados como alimento secundario, no encontrándose así alguna presa de tipo preferencial, por lo que no se considera a esta especie como un organismo especialista en su alimentación.

En conclusión, y según los resultados obtenidos de este trabajo y estudios que le anteceden, Prionotus ruscarius se encuentra en amplia variedad de hábitats, por lo que su conducta alimenticia adquiere un grado de complejidad, ya que se evidenció variaciones ontogenéticas y estacionales en la dieta de este pez.

\section{Agradecimientos}

Nuestro agradecimiento a los evaluadores anónimos por su valiosa contribución para mejorar esta publicación.

\section{Literatura citada}

Allen GR \& R Robertson. 1994. Fishes of the Tropical Eastern Pacific, 380 pp. University of Hawaii Press. Melbourne.

Bardach JE \& J Cse. 1965. Sensory capabilities of the modified fins of squirrel hake (Urophycis chuss) and sea robins (Prionotus carolinus and P. evolans). Copeia 1965(2): 194-200.

Braga FMS \& MAAS Braga. 1987. Estudo do hábito alimentar de Prionothus punctatus (Bloch, 1797) (Teleostei, Triglidae), na regiao da ilha anchieta, Estado de Sao Paulo, Brasil. Revista Brasileira de Biologia 47(1/ 2): 31-36.

Bocanegra-Castillo N, LA Abitia-Cárdenas \& F GalvánMagaña. 2000. Espectro alimentario de la berrugata californiana Menticirrhus undulatus de Laguna Ojo de Liebre, Baja California Sur, México. Ciencias Marinas 26(4): 659-675.

Bussing WA. 1995. Triglidae. En: Fisher W, F Krupp, W Schneider, C Sommer, KE Carpenter \& VH Niem (eds). Guía FAO para la identificación de especies para los fines de la pesca. Pacífico Centro-Oriental. Volumen III: 16431648.

Castro-Aguirre JL. 1978. Catálogo sistemático de los peces marinos que penetran a las aguas continentales de México con aspectos zoogeográficos y ecológicos. Departamento de Pesca, Instituto Nacional de Pesca (México). Serie Científica 19: 1-298.

Daniel WW. 1997. Bioestadística. Base para el análisis de las ciencias de la salud, 878 pp. Limusa Uteha Editorial Noriegal, México.

Fischer W, F Krupp, W Schneider, C Sommer, KE Carpenter \& VH Niem. 1995. Guía FAO para la identificación de especies para los fines de la pesca. Pacífico Centro-oriental. Volumen II. Vertebrados. Parte 1. Vol II: 647-1200.

Garth JS. 1958. Brachyura of the Pacific Coast of America: Oxyrhyncha. Allan Hancock Pacific Expeditions 21, Part 1-2: 1-854.

Gerking Shelby D. 1994. Feeding ecology of fish. 415 pp. Academic Press, San Diego.

Hacunda JS. 1981. Trophic relationships among demersal fishes in a coastal area of the Gulf of Maine. Fishery Bulletin 79(4): 775-788.

Hendrickx ME \& J Salgado-Barragán. 1991. Los estomatópodos (Crustacea: Hoplocarida) del Pacífico Mexicano. Instituto de Ciencias del Mar y Limnología. Universidad Nacional Autónoma de México 10: 1-200.

Hendrickx ME. 1996. Los camarones Peneiodea bentónicos (Crustacea: Decapoda: Dendrobranchiata) del Pacífico 
Mexicano. CONABIO. Instituto de Ciencias del Mar y Limnología. Universidad Nacional Autónoma de México. 155 pp.

Hespenheide HA. 1975. Prey characteristics and predator niche width. En: Cody ML \& JM Dinamond (eds). Ecology and Evolution of Communities, pp. 158-180. Belknap Press, Cambridge.

Jordan DS \& BW Evermann. 1896-1900. The fishes of North and Middle America. Bulletin of the United States National Museum 47(1-4): 1-3313.

Kouamélan PE, GG Teugels, G Gourène, DFE Thys van den Audenaerde \& F Ollevier. 2000. Habitudes alimentaires de Mormyrops anguilloides (Mormyridae) en milieux lacustre et fluvial d'un basin Ouest-African. Cybium 24(1): 67-79.

Krebs CJ. 1989. Ecological methodology. 550 pp. Harper \& Row, New York.

Lagler KF, J Bardach, R Millar \& D May Pasino. 1984. Ictiología. 489 pp. AGT, México.

Landa-Jaime V, J Arciniega-Flores, R García de QuevedoMachain, E Michel-Morfin \& G González-Sansón. 1997. Crustáceos decápodos y estomatópodos de fondos blandos de la plataforma continental de Jalisco y Colima, México. Ciencias Marinas 23(4): 403-417.

Lauzanne L. 1975. Régime alimentaire d'Hidrocyon forskali (Pisces: Characidae) dans le lac Tchad et ses tributaires. Cahiers ORSTOM, Série Hydrobiologie 9(2): 105-121.

Levins R. 1968. The theory of the niche. En: MacArthur RH (ed). Evolution in changing environments: Some theoretical explorations, pp. 39-66. Princeton University Press, Princeton.

Mariscal-Romero J, B Aguilar-Palomino, G LucanoRamírez, AR Raymundo-Huizar, LE Rodríguez-Ibarra, S Ruíz-Ramírez \& G González-Sansón. 1998. Asociaciones de peces demersales de la plataforma continental de Colima y Jalisco, México (Primavera, 1995). Ciencias Marinas 24(1): 35-54.

Moreno Amich R. 1988. Ecología trófica a la costa catalana i morfología alimentaria de la familia Triglidae (Pisces: Scorpaeniformes).Tesis Doctoral. Universidad Autónoma. Barcelona, Facultad de Ciències. Bellaterra. 334 pp.

Nikolsky GV. 1963. The ecology of fishes. 352 pp. Academic Press, New York.

Pinkas L, MS Oliphant \& ILK Inverson. 1971. Food habits of albacore, bluefin tuna and bonito in California waters.
Fish Bulletin 152: 1-105.

Rathbun MJ. 1930. The Cancroid crabs of America of the Families Eurylidae, Portunidae, Atelecyclidae, Cangridae an Xanthidae. Bulletin of the United States National Museum (152): 1-229.

Reid G K Jr. 1954. An ecological study of the Gulf of Mexico fishes, in the vicinity of Cedar Key, Florida. Bulletin of Marine Science Gulf Caribbean 4: 1-94.

Rodríguez de la Cruz MC. 1987. Crustáceos decápodos del Golfo de California. Secretaría de Pesca (Ed) México. 306 p. Parte 2 / 2: 145-306.

Ross ST. 1977. Patterns of resource partitioning in searobins (Pisces: Trigliade). Copeia 1977(3): 561-571.

Ross ST. 1978. Trophic ontogeny of the Leopard searobin, Prionotus scitulus (Pisces: Triglidae). Fishery Bulletin 76(1): 225-234.

Rosecchi E \& Y Nouaze. 1987. Comparaison de cinq indices alimentaires utilisés dans l'analyse des contenus stomacaux. Revues des Travaux de I'Institut des Pêches Maritimes 49: 11-123.

Schmitter-Soto JJ. 1992. Aspectos autoecológicos de los Triglidae (Pisces: Scorpaeniformes) en la costa occidental de Baja California Sur, México. Tesis de Maestría. Centro Interdisciplinario de Ciencias Marinas, Instituto Politécnico Nacional, La Paz, México, 102 pp.

Schmitter-Soto JJ \& JL Castro-Aguirre. 1996. Trophic comparison among Triglidae (Pisces: Scorpaeniformes) off Baja California Sur, México. Revista de Biología Tropical 44(2): 803-811.

Springer VG \& KD Woodburn. 1960. An ecological study of the fishes of the Tampa Bay area. Professional papers of the Florida State Board of Conservation: 1-104 pp.

Sturges H. 1926. The choice of a class-interval. Journal of the American Statistical Association (21): 65-66.

Thomson DA, LT Findley \& NA Kerstitch. 1979. Reef Fishes of the Sea of Cortez. 302 pp. John Wiley and Sons, New York.

Wooton RJ. 1990. Ecology of teleost fishes. 404 pp. Champman and Hall, New York.

Yañez-Arancibia A. 1978. Patrones ecológicos y variación cíclica de la estructura trófica de las comunidades nectónicas en lagunas costeras del Pacífico de México. Anales del Centro de Ciencias del Mar y Limnología. Universidad Autónoma de México 5 (1): 287-306. 